\title{
Dynamic response of auxetic structures
}

\author{
Matthieu Proffit ${ }^{1}$, John Kennedy ${ }^{2}$ \\ Trinity College Dublin, The University of Dublin, Dublin, Ireland \\ ${ }^{2}$ Corresponding author \\ E-mail: ${ }^{1}$ proffitm@tcd.ie, ${ }^{2} j k e n n e d 5 @ t c d . i e$
}

Received 29 April 2020; accepted 5 May 2020

DOI https://doi.org/10.21595/vp.2020.21440

Check for updates

Copyright (C) 2020 Matthieu Proffit, et al. This is an open access article distributed under the Creative Commons Attribution License, which permits unrestricted use, distribution, and reproduction in any medium, provided the original work is properly cited.

\begin{abstract}
Auxetic materials have been investigated since the 1990's, they are defined by a negative Poisson's ratio which gives them interesting capabilities in energy and shock absorption. They are usually lattice structures (2D or 3D array of unit cells), which gives them high stiffness to weight properties and their properties can be tuned to suit a given application. Several papers have been published on new auxetic designs, their static equivalent mechanical properties, shock absorption properties and their manufacturing. Their dynamic behavior has not been studied to date and this is the main purpose of this paper. We will present a novel design that facilitates 3D printing with SLA printers and then examine the dynamic behavior of an auxetic structure between 0 and $1 \mathrm{kHz}$. In order to explain the unusual behavior of the structure, an initial theoretical model of non-linear spring has been developed with partial success.
\end{abstract}

Keywords: auxetic, structures, dynamic, vibrations, frequency response, metamaterial, 3D printing, novel design, non-linear spring.

\section{Introduction}

Auxetic materials are materials that have a negative Poisson's ratio (they contract or expand in any spatial direction when exposed to a stress), they almost not be found in nature and their behavior emerges from their macro-structure. On a microscopic point of view, they are made from a bulk material of positive Poisson's ratio.

They have been studied since the late 1980s [1] for their good properties in shock absorption, fracture toughness, indentation resistance and energy dissipation. The auxetic properties often emerge from acute angles in a lattice structure (re-entrant hexagon for example) or spiral geometry.

The geometry presented in this paper is the hexagonal re-entrant structure, one of the most commonly studied [2-7] as it can be defined with very few parameters unlike other more complex structures. This geometry was first invented in 2D but with the development of additive manufacturing was extended to 3D. Samples studied in the literature were usually produced using SLM $[8,9]$ or other powder bed fusion methods, this produced good quality samples, but at high cost. The advantage of powder bed fusion methods is that the powder supports the structure as it is printed.

The current literature focuses on prediction of the structure's stiffness, the energy absorption [10], fracture toughness [11] and quasi-static properties [8]. To the author's knowledge, no study has been proposed that investigates their dynamic response.

This paper will therefore propose modifications on the lattice design to make it easily $3 \mathrm{D}$ printable with SLA printers (photo-sensible polymer resin that hardens with UV light). Enabling 3D printing with SLA printers may accelerate research on those structures as samples can be obtained for a very low cost ( $300 \$$ printers / $60 \$$ per liter of resin) and manufactured in any laboratory without specific investment. Their static properties have been investigated and compared to analytical models to assess the generalizability of results. The first requirement to enable SLA based printing methods is that the lattice is self-supporting during manufacture.

We will present a dynamic study of this auxetic structure, studying the influence of two parameters on the lattice frequency response function (FRF): the strut diameter and the mass 
attached to the sample. Dynamic results are a novelty for auxetic structures so we will initially attempt to use known analytical models to explain the structure's behavior.

\section{Novel structure}

This design varies from the usual design used in previous studies as it has been developed to be 3D printed on an SLA printer. The vertical beams have been modified and are now not flat as shown in Fig. 1.

Thus, they will not be printed in one layer, reducing the overhanging section, a recurrent problem for SLA printers (a non-self-supportive printing technique unlike SLS or SLM). The standard strut design was rectangular on most hexagonal re-entrant structures [12], this novel structure has cylindrical beams which reduces the number of parameters required to fully describe the structure. The vertical beam modification is done via the addition of another beam between the cell corner and a 16th of the angled beam.

As a side effect of the horizontal edge's modification, the beams junctions are now stronger (the horizontal struts are thicker at their ends). The stress concentrations located at the beam junctions pointed out by Carneiro et al. [13] by an FEA analysis of a 2D hexagonal re-entrant structure can be reduced [4]. This may be an interesting feature to enhance the lattice stiffness and fatigue strength without adding much material and complexity. It does also enable the production of the hexagonal re-entrant structure by SLA printers which is a major improvement as it drastically reduces the cost of production and may help further research on this topic.

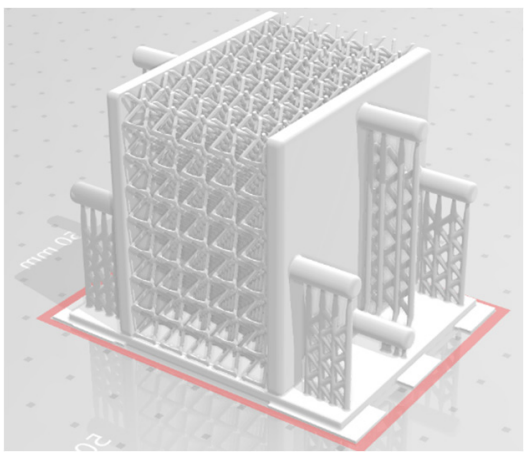

a)

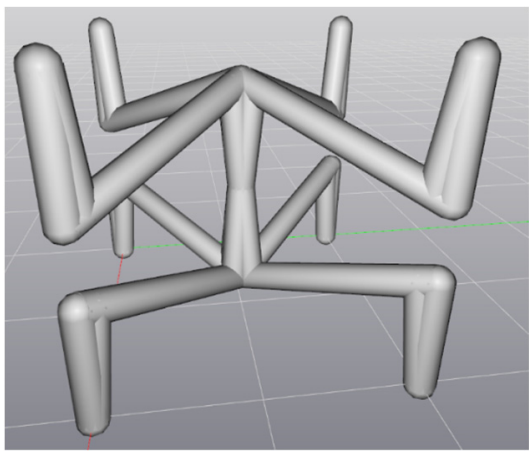

b)

Fig. 1. Novel lattice design for SLA printing: a) full sample with support, b) single cell

\section{Methodology - experimental procedure}

Several experimental procedures have been used, two different methods have been developed to measure the frequency response function (FRF) and make sure they were consistent, other tests have been conducted in a tensile/compression bench to evaluate the mechanical properties of the bulk material and the lattice stiffness.

\subsection{Dynamic experiments}

The measurements are performed under dynamic loading, the sample is attached by one end to a shaker and a mass $(m=0.124 \mathrm{~kg})$ is fixed to the other end. The output is measured by a laser vibrometer, the Polytech PDV100, that measures velocity with high accuracy. Both apparatuses are controlled via MATLAB and a NI-4431 acquisition card, the input is generated by MATLAB and converted by the acquisition card into a variable voltage. The output variable is the free end velocity of the sample, there is no need to integrate this variable in order to get the displacement as this would only result in a $90^{\circ}$ phase in the Fourier domain. The sampling rate is set at $48000 \mathrm{~Hz}$, the Fourier spectrum is however only exploitable up to $4 \mathrm{kHz}$ at best due to noise in the 
measurement.

The transfer function of the shaker is evaluated in a separate experiment and used to normalize the measured FRF and account for system performance.

The first method uses a white noise as input, the normalized output's Fourier transform is then compared to the input: the ratio of the two spectrum is the FRF of the sample. This method is the most widely used in this paper as it gives a very clean spectrum between 0 and $1 \mathrm{kHz}$, the noise starts to dominate at high frequencies.

The second method uses a sinusoid input and goes through the desired frequency range step by step. The amplitude of the output is then filtered and normalized by the same procedure as above (a reference measurement was conducted without sample) and plotted. This measurement method takes longer than the "noise measurement" as each frequency is tested one by one but enables an arbitrarily high frequency resolution.

\subsection{Quasi static tests}

Compression tests have been performed on the lattice to measure their stiffness, they are performed using an INSTRON 3366, the testing speed is $1 \mathrm{~mm} / \mathrm{min}$. All samples from a strut diameter of $0.5 \mathrm{~mm}$ to $1.2 \mathrm{~mm}$ have been tested three times in order to quantify the experimental uncertainty. Only the linear region of the stress-strain curve is considered, the tests are not destructive: they are stopped before the deformation becomes plastic (load lower than $50 \mathrm{~N})$. As the deformation is small enough and the stiffness is calculated in a linear regime, we can assume the tensile modulus is similar to the compressive modulus.

\section{Results}

\subsection{Static tests}

We compared the experimental results to two theoretical models developed by Yang et al. [14] and Shokri et al. [15] that predict the equivalent tensile modulus of an hexagonal re-entrant lattice. The first assumes only two modes of deformation are significant: it uses a Timoshenko beam model to describe the angled beam deformation and a classic compression model for the vertical beam. The second author uses Castigliano's theorem to calculate the tensile properties, the change in strain energy caused by a force divided by the force itself is equal to the displacement. Note that both methods need to be adapted for cylindrical beams (different cross section area, moment of inertia and Timoshenko beam factor). Although the two approaches are radically different, they give almost similar results as seen in Fig. 2. The novel hexagonal re-entrant structure is comparable to previously studied designs: its mechanical properties can be predicted from the analytical models developed for previous designs.

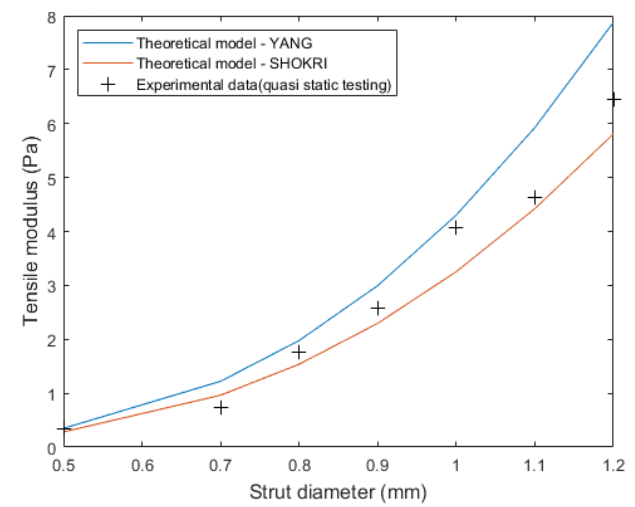

Fig. 2. Compressive tests results comparison to two analytical models 


\subsection{Dynamic experience - strut diameter influence}

From the range of samples produced ( 7 samples from $0.5 \mathrm{~mm}$ strut diameter to $1.2 \mathrm{~mm}$ ), we observed the FRF and compared them, the results are shown Fig. 3. The frequency response of an auxetic sample has a clear resonance frequency that can be observed in both the amplitude and phase spectrum. The peak width varies linearly with the peak frequency and their width at $-3 \mathrm{~dB}$ is on average $32 \%$ of the peak frequency.

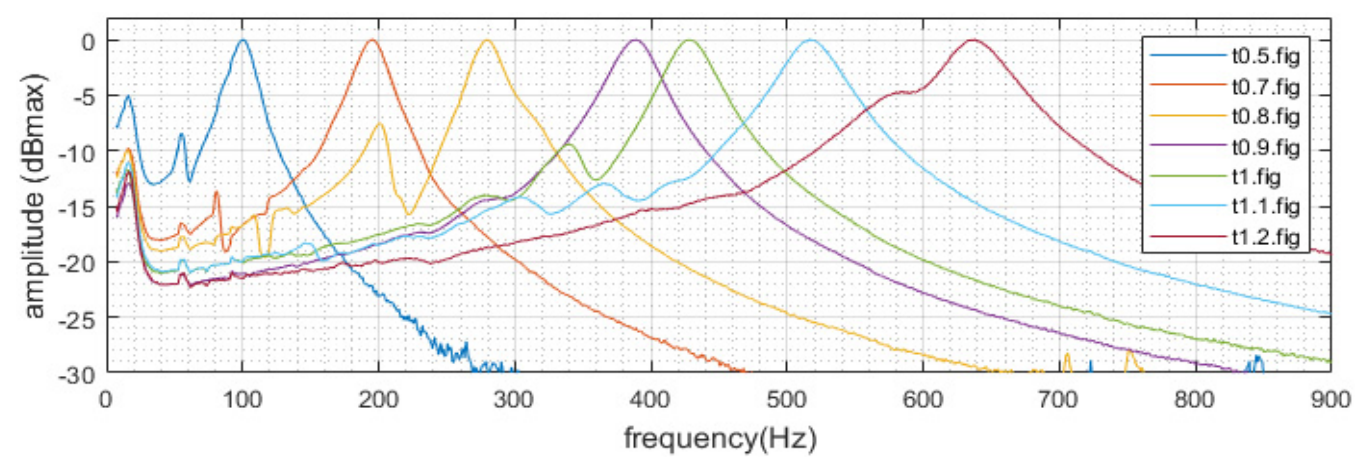

Fig. 3. Strut diameter influence on the FRF

We can see that each sample has a distinct peak corresponding to a resonance phenomenon in the structure, the stiffer the structure, the higher the resonance frequency. We can plot the resonance frequency VS strut diameter as shown in Fig. 4 to see quantitively how the strut diameter affects the dynamic behavior of the structure. We can see a clear linear trend between the resonance frequency and the strut diameter.

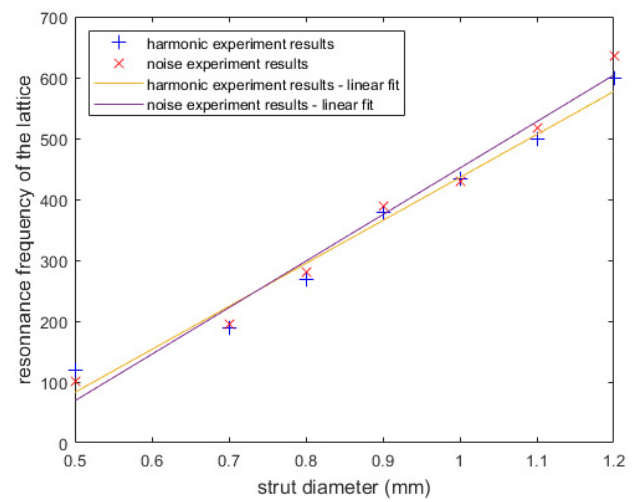

Fig. 4. Strut diameter influence on the resonance frequency

We can first try to explain that trend using a usual linear spring-mass model. As the resonance frequency is $f_{\text {res }}=\sqrt{k / m}$ if $k \sim t^{2}$, we find $f_{\text {res }} \sim t$. The second equation can be easily justified as the strut cross sectional area is proportional to $t^{2}$. This suggests the structure works more in beam compression than in bending (moment of inertia of a beam is proportional to $t^{4}$ ). This consideration is however incorrect as Yang et.al analytical model suggests the beam compression is only responsible for 5 to $25 \%$ of the lattice deformation $(5 \%$ for $d=0.5 \mathrm{~mm}, 25 \%$ for $d=1.2 \mathrm{~mm}$ ).

Furthermore, if we calculate $F=\sqrt{k / m}$ with $k$ being the measured static stiffness, we find the right trend but there is a factor $\sim 2$ between the calculated resonance frequency and the dynamic experimental results. 


\subsection{Mass variation}

As we saw previously, the linear spring mass model cannot explain the diameter-resonance frequency relationship. We varied the stiffness and observed a discrepancy in the results, in this part, we varied the mass and observed the variation in resonance frequency. Results are presented in Fig. 5 along with the predictions of a non-linear model which we will discuss in the next section. The experiments were conducted for various masses between $124 \mathrm{~g}$ (fixation weight) and about $500 \mathrm{~g}$ (maximum allowed weight for the shaker).

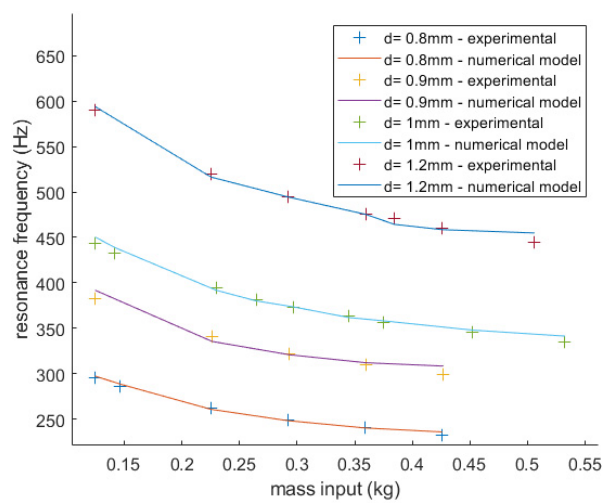

Fig. 5. Mass variation results and non-linear spring model predictions

\section{Discussion}

The results obtained in section 3.3 cannot be explained by a linear mass-spring model: the variation in resonance frequency does not correspond to any possible prediction of this model if the stiffness is kept constant (for the same sample). If we calculate a "virtual stiffness" for each experiment using $k=f^{2} m$ there is a clear linear relation between this fictional parameter and the mass (with 0 intercept). There is however a model that explains those unusual results almost perfectly as seen in Fig. 5, it is based on a non-linear spring with governing equation $F=\operatorname{sign}(\Delta) k^{\prime} \Delta^{4}$. The sign( ) function here is used to force $F(\Delta)$ to be odd, this equation is therefore not defined the same way for positive and negative deflections. This is one of the reasons why it is simply not possible to solve the governing differential equation of the mass-spring system, the only way to find the resonance frequency is by solving it numerically. We used the ode45() function on MATLAB to simulate the behavior of this non-linear system with 0 -initial deflection and acceleration and 1-initial velocity. It is worth noticing that such a system has an unual behavior and its resonance frequency varies with the initial velocity, the calculated stiffness k' has therefore no physical sense here as it has been arbitrarily set by choosing an initial velocity of 1 (setting one will force the other parameter during the optimization process to fit the results). To our knowledge there is no theoretical justification that the lattice material behaves like a non-linear material, especially for very small amplitude vibrations. As we saw, this model has however a great predictive power, this unusual dynamic behavior may be due to dissipative effects in the structure's bulk material that could be non-linear (a viscoelastic model could maybe be used to describe the resin dynamic behavior).

\section{Conclusions}

A novel structure has been developed with success, it enables 3D printing with SLA printers and has mechanical properties comparable to previously developed structures. It can be described by very few parameters: strut diameter, cell size (the cell fills a cubic volume here, so a single parameter is needed), vertical length ratio and additional strut location parameter (1/16 here) are 
sufficient to fully describe our novel design. It has been experimentally verified that analytical models developed for previous structures also predict the mechanical properties of our design with good accuracy.

Two dynamic methods have been used to determine FRF of auxetic samples: they give similar amplitude spectrum for different samples which validates them. The results obtained with those method cannot be explained with a simple mass-spring system (sample acting as a linear spring): even though the global trend of the linear system prediction is similar to the of the experimental results, there is an important multiplicative factor between both methods. Furthermore, when we vary the mass attached to the free end of the sample, the change in resonance frequency is not explained by a linear spring model. Even though there is no theoretical justification to the use of a polynomial spring model, it happens that a non-linear spring with governing equation $F=\operatorname{sign}(\Delta) k \Delta^{4}$ explains almost perfectly the experimental results. Dissipative effects inside the resin that are not considered in the theoretical models may play a significant role in the dynamic behavior of the sample and be absolutely negligible in quasi-static behavior. This would have to be verified in further studies, which would be highly facilitated by the use of SLA printed samples, which are much easier to produce in significant quantities in a relatively short time and at a moderate cost.

\section{References}

[1] Almgren R. F. An isotropic three-dimensional structure with Poisson's ratio $=-1$. Journal of Elasticity, Vol. 15, Issue 4, 1985, p. 427-430.

[2] Masters I. G., Evans K. E. Models for the elastic deformation of honeycombs. Composite Structures, Vol. 35, Issue 4, 1996, p. 403-422.

[3] Grima J., Oliveri L., Attard D., Ellul B., Gatt R., Cicala G., et al. Hexagonal honeycombs with zero Poisson's ratios and enhanced stiffness. Advanced Engineering Materials, Vol. 12, Issue 9, 2010 , p. $855-862$.

[4] Cirone S., Hayes G., Babcox B., Frecker M., Adair J., Lesieutre G. Design of contact-aided compliant cellular mechanisms with curved walls. Journal of Intelligent Material Systems and Structures, Vol. 23, Issue 16, 2012, p. 1773-1785.

[5] Chen Y., Fu M.-H. A novel three-dimensional auxetic lattice meta-material with enhanced stiffness. Smart Materials and Structures, Vol. 26, Issue 10, 2017, p. 105029.

[6] Grima J. N., Gatt R., Alderson A., Evans K. E. On the potential of connected stars as auxetic systems. Molecular Simulation, Vol. 31, Issue 13, 2005, p. 925-935.

[7] Kim K., Ju J., Kim D.-M. Cellular materials with extremely high negative and positive Poisson's ratios: a mechanism based material design. International Mechanical Engineering Congress and Exposition, 2013.

[8] Yang L., Harrysson O., Cormier D., West H., Park C., Peters K. Design of auxetic sandwich panels for structural applications. Annual International Solid Freeform Fabrication (SFF) Symposium, 2013.

[9] Yan C., Hao L., Hussein A., Raymont D. Evaluations of cellular lattice structures manufactured using selective laser melting. International Journal of Machine Tools and Manufacture, Vol. 62, 2012, p. 32-38.

[10] Yazdani Sarvestani H., Akbarzadeh Ah, Mirbolghasemi A., Hermenean K. 3D printed meta-sandwich structures: Failure mechanism, energy absorption and multi-hit capability. Materials and Design, Vol. 160, 2018, p. 179-193.

[11] Choi J.-B., Lakes R. Fracture toughness of re-entrant foam materials with a negative Poisson's ratio: experiment and analysis. International Journal of Fracture, Vol. 80, 1996, p. 173-183.

[12] Saxena K. K., Das R., Calius E. P. Three decades of auxetics research - materials with negative Poisson's ratio: a review. Advanced Engineering Materials, Vol. 18, Issue 11, 2016, p. 1847-1870.

[13] Carneiro V., Meireles J. Elasto-plastic shear behavior of reinforced honeycomb and auxetic reentrant lattices. Millenium, Vol. 7, Issue 2, 2018, p. 81-90.

[14] Yang L., Harrysson O., West H., Cormier D. Modeling of uniaxial compression in a 3D periodic re-entrant lattice structure. Journal of Material Science, Vol. 48, Issue 4, 2013, p. 1413-1422.

[15] Shokri Rad M., Prawoto Y., Ahmad Z. Analytical solution and finite element approach to the 3D re-entrant structures of auxetic materials. Mechanics of Materials, Vol. 74, 2014, p. 76-87. 